\title{
Computerized games to study the development of attention in childhood
}

\author{
ANDREA BERGER \\ Ben-Gurion University of the Negev, Beer-Sheva, Israel \\ and Sackler Institute for Developmental Psychobiology, Weill Medical College \\ Cornell University, New York, New York \\ LAURA JONES \\ University of Oregon, Eugene, Oregon \\ and \\ MARY K. ROTHBART and MICHAEL I. POSNER \\ University of Oregon, Eugene, Oregon \\ and Sackler Institute for Developmental Psychobiology, Weill Medical College \\ Cornell University, New York, New York
}

\begin{abstract}
Children enjoy playing games. We can take advantage of this in the designs of computerized tasks that will engage their interest. These designs also serve to advance the study of chronometric measures, such as manual and saccadic reaction times and event related potentials, with young children. The goals of our method development are (1) to allow for comparable tasks across a wide variety of ages, (2) to make possible comparisons of child performance with data gathered in adult cognitive studies, and (3) to help to support inferences about the development of underlying mechanisms. We have designed a battery of computerized tasks in order to study the development of attention functions of alertness, orienting, and executive control during childhood. Our purpose is to describe each of these tasks in detail and present the results that have been obtained so far. The battery was tested using a sample of 5-year-old children as subjects.
\end{abstract}

Posner and Petersen (1990) identified three attentional networks, each one having a unique function and a specified neuroanatomical basis. Posner and Raichle (1996) summarized the three attentional networks' localization in the brain, on the basis of behavioral, neuropsychological, and brain imaging data. (1) The orienting network is responsible for focusing, disengaging, and shifting of spatial attention. These attentional operations involve a brain network including the posterior parietal lobes, the pulvinar nucleus of the thalamus and the superior colliculus. (2) The vigilance network is responsible for maintenance of an alert state. This function seems to involve the right lateralized parietal and right frontal cortical networks and also the locus coruleus. (3) The executive network is responsible for goal-directed behavior, target detection, error detection, conflict resolution and inhibition of automatic responses. The executive network seems to include the midline frontal areas including the anterior cingulate gyrus, supplementary motor area, and portions of the basal ganglia.

This work has been partially supported by a fellowship of the Yad Hanadiv-The Rothschild Foundation given to the first author. The programs described in this paper can be freely provided for research purposes. However, we are not able to provide support or to guarantee that they will work with different hardware equipment, especially since touch sereens tend to differ. Correspondence should be addressed to A. Berger, Behavioral Science Department, Ben-Gurion University of the Negev, Beer-Sheva 84105. Israel (e-mail: andrea( $\omega$ bgumail.bgu.ac.il).
Each attentional network has been studied in the literature using a particular paradigm that seems to serve as a marker task for that specific functional network. These paradigms are mostly based on manual responses and reaction time (RT) measurements. They have been developed for use with adult subjects (normal and neurological patient populations).

All three networks of attention seem to undergo intense postnatal development (Ruff \& Rothbart, 1996), as their underlying neuroanatomical structures mature. To trace this development, there is a need for a suitable methodology. However, the adult paradigms usually include hundreds of trials and extremely simple and boring stimuli (mostly asterisks) and are not suitable for very young subjects.

The challenge is to find a way to (1) allow for comparable tasks across a wide variety of ages, (2) make possible comparisons of children's performance with data gathered in adult cognitive studies, and (3) help to support inferences about the development of underlying mechanisms. The task is much easier for children of school ages, since most of the common adult cognitive paradigms are also suitable for them (see, e.g., Enns, Brodeur, \& Trick, 1998).

For very young ages, on the other hand, paradigms based on eye movements (Clohessy, Posner, \& Rothbart, \& Vecera, 1991; Hood, Atkinson, \& Braddick, 1998; Johnson, Posner, \& Rothbart, 1991; Maurer \& Lewis, 1998; Pos- 
ner \& Rothbart, 1994) and physiological responses, such as changes in heart rates and respiratory sinus arrhythmia (Richards \& Hunter, 1998), have been developed.

In toddlers and children of preschool age, motor skills and hand-eye coordination enable the measurement of manual RTs, allowing the comparison with older children and adults. However, these responses can be collected only if we succeed in engaging the child's attention and as long as the child stays "on task." A feasible way to do this is to take advantage of what children like most-that is, to play games. Our idea was to develop a series of computerized game-like tasks, strictly based on the attention paradigms from the adult literature. Each task is presented to the subject as a story in which the child's responses to stimuli are an integral part. The alert task is designed to tap the alert and arousal network, measuring the phasic alertness induced by a warning signal, and is presented to the child as the "help the farmer" game. The orienting task, which is an adaptation of Posner's cuing paradigm for spatial orienting, is designed to tap the orienting of spatial attention induced by an exogenous peripheral cue and is presented to the child as the "feed the fish" game. In the spatial conflict task and the pointing-Stroop task, the child is required to resolve a cognitive conflict, in order to tap the function of executive attention. The spatial conflict task is presented to the child as the "help the pictures find their home" game, and the pointing-Stroop task is presented as the "animals and their voices" game.

The games are run on a computer, using a touch-screen monitor for stimulus display and response collection (see details in the Apparatus section). As mentioned, the games were designed on the basis of RT paradigms that have been used extensively in cognitive psychology and neuropsychology for studying the different functions of attention.

We now present detailed descriptions of the tasks and the results that have been obtained using a sample of 5year-old children.

\section{METHOD}

\section{The Games}

Alert task. The alert task measures change in the internal state (phasic arousal) following the presentation of a warning signal. The task is presented to the child as a "help the farmer catch the animals that want to run away" game, in which different farm animals (targets) appear on the screen, one at a time. When the child touches the animal, it "goes back to the farm" and disappears from the screen. At the end of the game, the child is presented with a picture of the farm with all the animals that the child helped to catch.

The warning signal is a tone presented at different intervals before the target's appearance. ${ }^{1}$ RTs to tone and no-tone trials reflect the influence of the warning signal. The rate of change in RT at the different warning intervals measures the development of phasic alertness.

The targets are farm animal pictures of about $6^{\circ} \times 6^{\circ}$. They are presented one by one at the center of the screen. The warning signal (the tone) is presented in half of the trials before the target appears, at one of the following intervals: $200,500,1,000$ or 2,500 msec. ${ }^{2}$ From target onset, the subject has $10,000 \mathrm{msec}$ to respond. If the subject responds, the target disappears and the program immediately proceeds to the next trial. The intertrial interval (ITI) is
$1,000 \mathrm{msec}$. There are 8 experimental conditions in this task (tone/no-tone $\times 4$ intervals). The task itself consists of 32 trials, along with 4 practice trials at the beginning. It takes about $5 \mathrm{~min}$ to complete the whole task.

Orienting task. This task is adapted from Posner's cuing paradigm for spatial orienting of attention (Posner, 1978). Instead of the usual square boxes that appear on a screen, there are two glass tanks presented $10^{\circ}$ to the left and to the right of a fixation point. Instead of using an asterisk as a target, we use a fish that can appear in either one of the tanks ( $50 \%$ in each, randomly). The task is presented as a "feed the fish" game in which, each time a fish appears, the subject has to try to feed it by touching it with a finger as fast as possible (before the fish disappears). The child has a small worm on a finger that represents the symbolic food for the fish.

Trials consist of a fixation stimulus, followed by a cue, and then the target appears (i.e., a fish inside one of the tanks). The fish is presented on the screen for a maximum of $1,000 \mathrm{msec}$, as long as the child does not touch the fish. If the child touches the fish, it disappears and a positive feedback is given (the fish says, "thank you"). The cue consists of a color change of one of the tanks (from light blue to dark blue and back to light blue). Duration of the cue is $500 \mathrm{msec}$. Cues appear at each tank with equal probability. The interval between the beginning of the cue and the beginning of the target (stimulus onset asynchrony, SOA) is either 100 or $1,000 \mathrm{msec}$ ( $50 \%$ each, randomly selected). The ITI is $1,000 \mathrm{msec}$.

There are 4 practice trials at the beginning of the experiment. The task itself consists of 32 trials ( 4 trials for each combination of cue location, target location, and SOA) and takes about $5 \mathrm{~min}$ to complete.

Spatial conflict task. The spatial conflict task (Gerardi,1997) is presented to the child as a "help the picture find its home" game and consists of a matching game in which, half of the time, there is a conflict between the location of the stimulus and the response. The task objective is to measure the interference effects, in RTs and accuracy, created by the incompatibility between stimulus and response spatial locations. This is a nonverbal measure of executive attention, since the child must inhibit a prepotent response to comply with task demands. Children attend to a stimulus in one spatial location, but they are required to respond to the opposite location. The tendency to respond to the same side in which the target appears causes even adults to slow down their RTs in the incompatible trials (Simon, 1990). Gerardi (1997) found developmental improvement is RTs and accuracy in toddlers 24-38 months of age.

At the beginning of each trial, two houses appear on the left and right bottom corners of the screen, with a picture inside each house. A central looming lasts until the experimenter is sure that the child's attention is focused on the screen, after a picture appears in either the left upper corner or the right upper corner. If the picture appears on the same side as the corresponding inside-the-house picture, it is a compatible trial. If the picture is on the opposite side, it is an incompatible trial.

The child's job is to match the pictures "to help the picture find its home," by touching the corresponding house. To succeed, the child needs to ignore the spatial location of the picture, which, half of the time, is located opposite to the correct response. The picture stays on the screen until the child's response or a maximum of $15 \mathrm{sec}$. After the response, there is a feedback sound (positive or negative). The positive sound is accompanied by a flickering of the picture. There are 2 experimental conditions in this task (spatially compatible and incompatible trials) presented in a random order. At the beginning of the task, there are 4 practice trials in which the picture appears in a neutral position (central upper position). The task consist of 32 trials and takes about $10 \mathrm{~min}$ to complete.

Pointing-Stroop task. The Stroop task (Stroop, 1935) is wellknown paradigm requiring the inhibition of a prepotent response. Several versions of the task have been used in the developmental literature (e.g., Gerstardt, Hong, \& Diamond, 1994; Jerger, Martin, \& Pirozzolo, 1988; Passler, Isaac, \& Hynd, 1985). Gerstardt et al. 

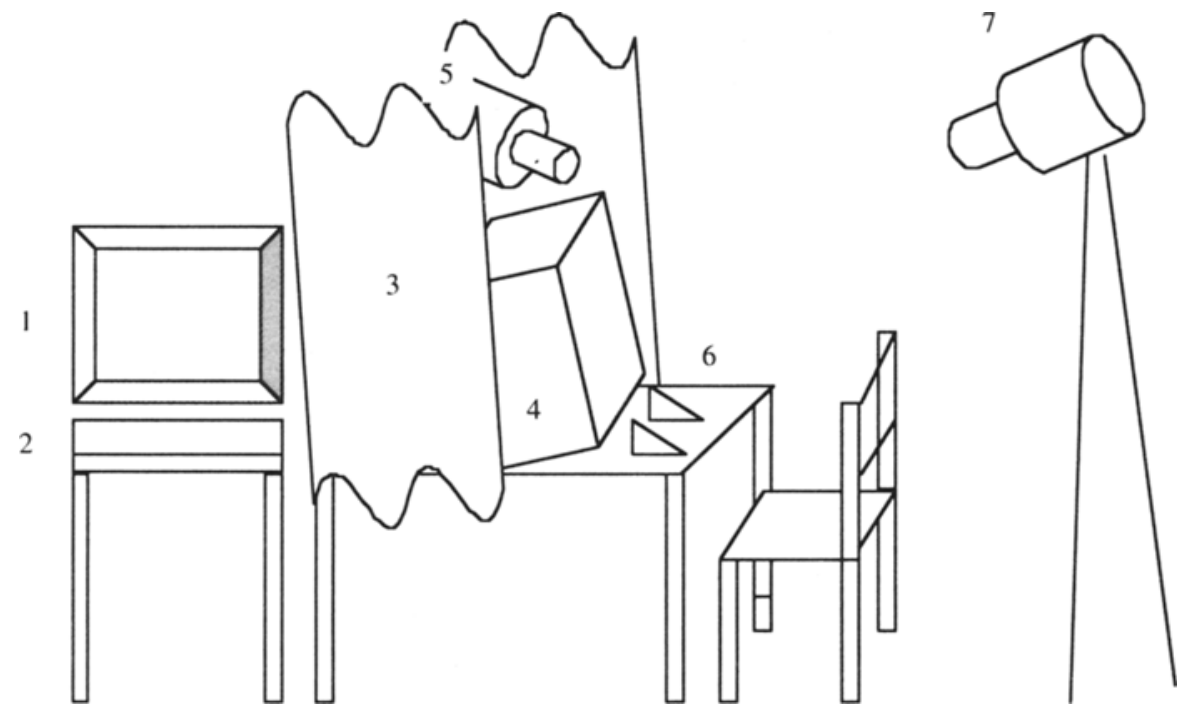

Figure 1. Schematic diagram of the apparatus setting: (1) experimenter's post (control monitor and keyboard), (2) videotape, (3) curtain, (4) touch screen, (5) camera $A$ focused on the child's face, (6) hand-position holders for the child, and (7) camera B focused on the child's hands and the stimulus on the screen.

(1994), for example, developed a Stroop-like test in which children were instructed to say "day" when presented with a black card showing a moon and stars and to say "night" when presented with a white card showing a sun. Carlson (1997) used a variation of this task, in which children responded by pointing instead of speaking. In this version of the task, the children were asked to point to a white card when they heard the word grass and to a green card when they heard the word snow. The pointing-Stroop task in this study was found to be a good index of individual differences in inhibitory control in preschool age children.

The present pointing-Stroop task is an auditory-visual computerized version of Carlson's pointing-Stroop task. In this task, two pictures of animals appear on the screen (e.g., a dog and a cat). An animal voice (the voice of one of the animals on the screen) is heard simultaneously (e.g., "meow"). In the compatible block ( 16 trials), the child points to (touches) the animal that makes the sound. If the child touches the correct animal, there is a positive feedback (the animal moves, and there is also a positive sound). Otherwise, an "error" feedback sound is given. After the compatible block, the child is told that he/she will now play a silly and funny game, in which he/she touches the opposite animal (e.g., if a cat and a dog are presented together with barking, the child should touch the cat). This is the incompatible block ( 16 trials).

In this task, it could be argued that there is a comprehension difficulty (especially in the incompatible blocks), so the child does not understand the instructions to touch the animal that "doesn't make the sound." Therefore, before the experimental trials begin, the experimenter uses a card on which there is a pair of pictures similar to the ones that will appear on the screen and practices the game with the subject. The experimenter makes sure that the child understands the game and is able to reproduce the required response.

It could also be argued that there is a memory difficulty, so that, after a short while, the child forgets the rules of the game. To avoid this problem, the experimenter reminds the child of the purpose of the game every 3 trials during the ITIs.

The size of each animal picture is about $6^{\circ} \times 6^{\circ}$. and all pictures are displayed along the horizontal meridian, $10^{\circ}$ to the left and right of fixation. The animal pair, sides of presentation, voices, and so forth are counterbalanced across the different trials and randomly selected.

There are 32 trials, 16 within each block. The task takes about 7 min to complete.

\section{Apparatus}

The subject sits in a high chair or booster seat at the wide end of a table. The chair is located approximately $13 \mathrm{in}$. from the monitor. The computer monitor ( $15.5 \times 11.5 \mathrm{in}$.) is a touch screen, specially designed to respond to touch. In front of the subject, there are two felt cut-outs in the shape of a child's hands, on which the subjects are instructed to place their hands between trials.

A curtain is draped between the experimenter and the subject. The curtain frames the touch-screen monitor and a camera lens. Two cameras are used in this procedure. One camera, located directly above the monitor, films the child's face and upper body. A second camera, located directly behind the subject, films the touchscreen monitor. The experimenter watches both views on a splitscreen monitor. Parents sit in a cubicle, out of their child's view, and watch the session on a television monitor. See Figure 1 for a diagram of the apparatus setting.

The following sections of the paper relate to the test carried out at the University of Oregon. using a sample of 5-year-old children.

\section{Subjects}

Thirty children ranging in age from 58 to 62 months $(M=60.3$ months) participated in the study. There were 15 females and 15 males. The subjects were recruited from the community, on the basis of birth announcements in the local newspaper. They were contacted by phone and asked to participate in the study. Participation was voluntary. As a token of our appreciation, each child received either a "University of Oregon Junior Scientist" T-shirt or a gift certificate from one of the local toy stores, depending on his/her choice.

\section{Procedure}

The children came to the laboratory for a 1-h session. After a short warm-up in the greeting room where they played with toys and became accustomed to the experimenter and the lab, they sat in 


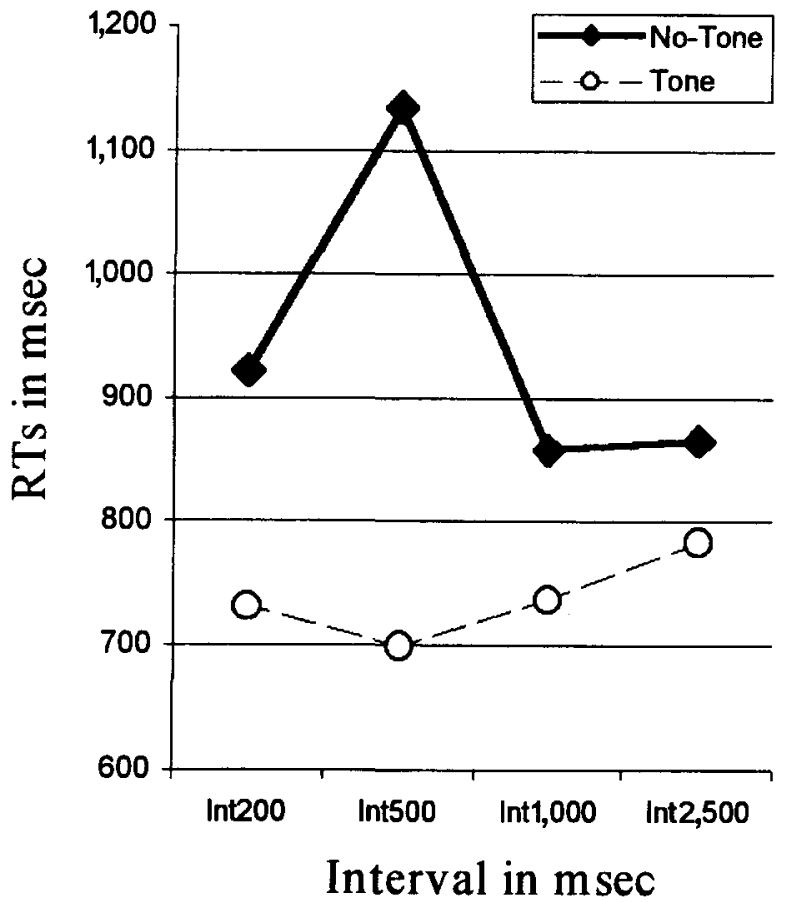

Figure 2. Reaction times in milliseconds in the alert task as a function of the tone condition (tone vs. no tone) and the interval $(200,500,1,000$, and 2,500 msec).

front of the touch-screen monitor and the session began. The first task was always the alert task; the second task was always the spatial conflict task. ${ }^{3}$ There was a 10 -min break during which the children colored a picture. In the second part of the session, the children played the pointing-Stroop and orienting games in random order.

The children were not restricted regarding which hand(s) to use for responses, in any of the games. However, to control for the trajectory length of the reaching motor response, they were instructed to place their hands on the felt hand holders between trials and to put their hands back on them immediately after each response. The experimenter monitored the children's hand placement and, if necessary, reminded them to go back to the hand holders.

At the beginning of each game, the experimenter explained the game, verifying that the children understood the purpose of the game during the practice trials. The experimenter then went back to a position behind the curtain and remained there monitoring the stimulus presentation for the rest of the trials. No verbal feedback was given during the trials.

In addition to the RTs and errors collected by the computer, the session was videotaped using two video cameras.

\section{General Analysis}

In our sample of 5-year-old subjects, the children made no errors in the games, and we therefore focused on the RT analysis. In a very small proportion of the trials (about $3 \%$ ), it happened that the contact between the child's finger and the touch-screen monitor was too weak to be detected, and the child had to touch the screen a second time. This led to misleadingly long RTs. Since the sessions were videotaped, these cases were localized, and RT was corrected, if possible, using the frame-by-frame analysis of the videotape or were otherwise discarded from the analysis.

Median RTs were calculated for each subject in each experimental condition. These medians were subjected to a repeated measures analysis of variance (ANOVA; the design was specific to each task described above).

\section{RESULTS AND DISCUSSION}

\section{Alert Task}

An ANOVA was performed using tone (tone and no tone) and interval $(200,500,1,000$, and $2,500 \mathrm{msec})$ as within-subjects variables. There was a significant main effect of tone $[F(1,13)=19.014, p<.001]$, with shorter RTs is tone trials than in no-tone trials. There was also an interaction between tone and interval $[F(3,39)=3.45$, $p<.05$ ] (see Figure 2).

Contrasts between tone and no-tone trials at the different intervals showed that the difference was significant only between the first two intervals [ 200 and $500 \mathrm{msec}$; $F(1,13)=5.05, p<.03$, and $F(1,13)=24.871, p<.0001$, respectively]. Moreover, the effect of the tone was significant even without including the 500 -msec interval $[F(1,13)=11.415, p<.004]$, for which there was an unexplained upswing in the no-tone graph.

It is interesting to compare these results with those we obtained using a sample of 12 undergraduate students. The adults showed a main effect of tone independently of the interval value $[F(1,11)=20.13, p<.001]$ and a main effect of interval $[F(3,33)=185.54, p<.0001]$, showing shorter and shorter RTs the longer the interval. This interval effect seems to reflect a well-known strategic component in the RT literature. When intervals are used with equal frequency, the longer the time that goes by since the warning or last keypress, the more certain the subject is that the next target is about to appear. No interaction between tone and interval effects was found in the adult sample.

Two differences in the results of the 5-year-old children and the adults should be pointed out. First, the alerting effect of a task-irrelevant tone seems to be in the same direction but less long lasting in the children than in the adults. Second, the strategic component is completely absent in the children's data. We are currently gathering data using a wider range of ages, since more sampling points seem to be necessary in order to understand these developmental processes and the maturation of phasic alertness.

\section{Orienting Task}

An ANOVA was performed using cue validity (valid and invalid) and SOA (100 and 1,000 msec) as withinsubjects variables. There was a significant main effect of cue validity $[F(1,29)=12.194, p<.01]$, showing shorter RTs in valid trials than in invalid trials. There was also a main effect of SOA $[F(1,29)=4.944, p<.5]$. The interaction between cue validity and SOA $[F(1,29)=9.844$, $p<.005$ ] showed that, at the short SOA, there was a significant cue effect $[F(1,29)=23, p<.0001]$, whereas at the long SOA, this effect was gone $(F<1)$ (see Figure 3 ). This pattern, even without a full crossover of the graph 


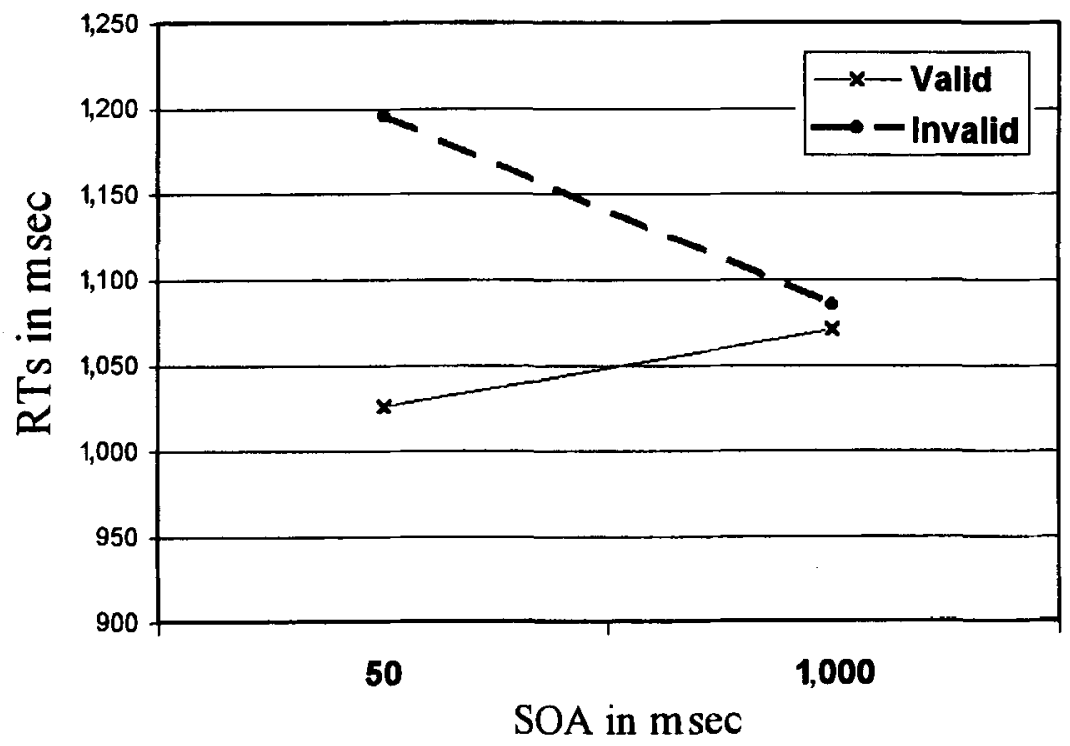

Figure 3. Reaction times in milliseconds in the orienting task as a function of the cuevalidity condition (valid vs. invalid) and the SOA (100 and $1,000 \mathrm{msec})$.

lines, seems to indicate the beginning of inhibition of return (IOR; this point will be discussed more extensively in the Discussion section). In fact, for $60 \%$ of the children in the sample, there was a full crossover of the lines, in which valid trials were slower than invalid trials.

IOR was first described by Posner and Cohen (1984) and has been extensively studied since then (see Rafal $\&$ Henik, 1994, and Taylor \& Klein, 1998, for a comprehensive summary of IOR research). It appears following an exogenous noninformative peripheral cue and appears to produce the inverse of the cuing effect, in which, at long SOAs, valid trials become slower than invalid trials. IOR has been observed in infants and even newborns, in studies measuring probability of saccadic eye movements toward the cued target versus the uncued target instead of the RT (Clohessy et al., 1991; Hood \& Atkinson, 1997; Simion, Valenza, Umiltà, \& Barba, 1995). Our task allows for a way to bridge the gap between these infant studies and the adult IOR literature, for toddlers and preschoolage children. School-age children, as mentioned, have been extensively studied using the standard cuing paradigm (Enns et al.,1998).

\section{Spatial Conflict Task}

A one-way ANOVA was performed with compatibility (compatible and incompatible) as the within-subjects variable. There was a significant main effect of compatibility $[F(1,29)=18.608, p<.0005]$, showing shorter RTs in compatible trials $(1,590 \mathrm{msec})$ than in incompatible trials $(1,706.5 \mathrm{msec})$.

The results are consistent with Gerardi (1997) and seem to extend and continue the trend found in her study, in the sense of improved accuracy and overall decreased RTs with age. In Gerardi's (1997, Study I) data, 24-monthold subjects performed at $63 \%$ and $53 \%$ accuracy for compatible and incompatible trials, respectively, and there was no effect in RTs (mean RT around 3,400 msec). The 30-month-old subjects performed at $78 \%$ and $61 \%$ accuracy and were also $271 \mathrm{msec}$ faster in compatible trials than in incompatible trials $(3,137$ vs. $3,408 \mathrm{msec})$. The 36-38-month-old subjects performed at $92 \%$ and $85 \%$ and were also $604 \mathrm{msec}$ faster in compatible trials than in incompatible trials $(2,476 \mathrm{vs} .3,079 \mathrm{msec})$. In our study, 60-month-old subjects were able to perform at $100 \%$ accuracy and were $116 \mathrm{msec}$ faster in compatible trials than in incompatible trials $(1,590$ vs. $1,706 \mathrm{msec})$. For children 5 years of age, the spatial conflict task seemed to be easy, and their accuracy was perfect. However, it still produced a small, but significant, compatibility effect in RTs.

\section{Pointing-Stroop Task}

A one-way ANOVA was performed with compatibility (compatible and incompatible) as the within-subjects variable. There was a significant main effect of compatibility $[F(1,29)=22.951, p<.0001]$, with shorter RTs in compatible trials $(1,419 \mathrm{msec})$ than in incompatible trials $(1,758 \mathrm{msec})$.

Since our subjects did not make errors, it was evident that they understood the task. Some of them were very amused by the idea of touching the "wrong" animalthat is, the one that did not make the sound. Moreover, it is unlikely that the longer RTs in the incompatible block were caused by a memory load due to the need to hold the purpose of the task in memory, since, as was explained earlier, the experimenter reminded the children of the instructions every 3 trials. It seems that the difference in mean RTs between the conditions was due to the need to ignore the irrelevant conflicting information for the auditory channel in the incompatible condition. This cog- 
nitive conflict seemed to be stronger and led to larger effects than the one between spatial location and identity presented in the spatial conflict task. ${ }^{4}$

In concurrence with Carlson's (1997) results, the size of the compatibility effect in this task was found to be negatively correlated with measures of effortful control as reported by the parents $(r=-.51, p<.05) .^{5}$

\section{Conclusions}

We developed a battery of tasks in which a standard paradigm from the cognitive literature is embedded in a game. We took advantage of children's love of and interest in play, and we created a computerized design to study cognitive processes using chronometrical measures of RTs. Our battery focused on marker tasks for the different networks and processes of attention.

At this point, the battery has been mainly tested with 5year-old children, leading to clear and measurable effects. The reaction of all our 5-year-old subjects to the games has been positive. Moreover, the children seem very fond of the games and enjoy the lab session.

The games are also suitable for a wider range of ages, from toddlers to school-age children. The alert task and the spatial conflict task are currently in a cross-sectional study at the University of Oregon for children 18,24 , and 36 months of age.

Due to the target ages of the battery, one of the biggest challenges was to obtain effects even with a very small number of trials. In comparison with the original paradigms from the cognitive literature, on which we based our tasks, the number of trials that we used was extremely low, with a ratio of about 1:10. Even under these limitations, the results show, so far, very strong and clear effects.

We believe that this battery of tasks can be very useful for the study of the developmental course of attention networks. Moreover, this methodology can be useful for comparing these processes between normal and special populations (e.g., children at risk of ADHD, prematurely born children, etc.) to track individual differences and to connect these differences to other individual characteristics, such as temperament.

\section{REFERENCES}

Berger, A., Jones, L., Rothbart, M. K., \& Posner, M. I. (2000). Alerting the executive attention system in five vear old children. Manuscript submitted for publication.

CARLSON, S. M. (1997). Individual differences in inhibitory control and children's theory of mind. Unpublished doctoral dissertation, University of Oregon.

Clohessy, A. B., Posner, M. I., Rothbart, M. K., \& Vercera, S. P. (1991). The development of inhibition of return in early infancy. Journal of Cognitive Neuroscience, 3, 345-350.

Enns, J. T., BrodeUr, D. A., \& Trick, L. M. (1998). Selective attention over the life span: Behavioral measures. In J. E. Richards (Ed.), Cognitive neuroscience of attention: A developmental perspective (pp. 393418). Mahwah, NJ: Erlbaum.

Gerardi, G. M. (1997). Development of executive attention and self- regulation in the third year of life. Unpublished doctoral dissertation, University of Oregon.

Gerstardt, C. L., Hong, Y. J., \& Diamond, A. (1994). The relationship between cognition and action: Performance of children 3.5-7 years old on a Stroop-like day-night test. Cognition, 53, 129-153.

HoOd, B., \& AtKinson, J. (1997). Inhibition of return in infants. Perception, $19,369$.

Hood, B. M., Atkinson, J., \& Braddick, O. J. (1998). Selection-foraction and the development of orienting and visual attention. In J. E. Richards (Ed.), Cognitive neuroscience of attention: A developmental perspective (pp. 219-250). Mahwah, NJ: Erlbaum.

Jerger, S., Martin, R. C., \& Pirozzolo, F. J. (1988). A developmental study of the auditory Stroop effect. Brain \& Language, 35, 86-104.

Johnson, M. H., Posner, M. I., \& Rothbart, M. K. (1991). Components of visual orienting in early infancy: Contingency learning, anticipatory looking, and disengaging. Journal of Cognitive Neuroscience, 3, 335-344.

MAURER, D., \& LEWIS, T. L. (1998). Overt orienting toward peripheral stimuli: normal development and underlying mechanisms. In J. E. Richards (Ed.), Cognitive neuroscience of attention: A developmental perspective (pp. 51-102). Mahwah, NJ: Erlbaum.

Passler, M. A., IsAac, W., \& Hynd, G. W. (1985). Neuropsychological development of behavior attributed to frontal lobe functioning in children. Developmental Neuropsychology, 1, 349-370.

POSNER, M. I. (1978). Chronometric explorations of the mind. Hillsdale. NJ: Erlbaum.

Posner, M. I., \& COHEN, Y. (1984). Components of visual orienting. In H. Bouma \& D. G. Bouwhuis (Eds.), Attention and performance $X$ : Control of language processes (pp. 531-556). Hillsdale, NJ: Erlbaum.

Posner, M. I., \& Petersen, S. E. (1990). The attention system of the human brain. Annual Review of Neuroscience, 13, 25-42.

Posner, M. I., \& Raichle, M. E. (1996). Images of mind (rev.). New York: Scientific American.

Posner, M. I., \& Rothbart, M. K. (1994). Constructing neuronal theories of mind. In C. Koch \& J. Davis (Eds.), High level neuronal theories of the brain (pp. 183-199). Cambridge, MA: MIT Press.

Rafal, R., \& HeNIK, A. (1994). Inhibitory neural mechanisms for integrating controlled and automatic processes. In D. Dagenbach \& T. H. Carr (Eds.), Inhibitory processes in attention, memory, and language (pp. 1-51). San Diego: Academic Press.

RichaRDS, J. E., \& HUNTER, S. K. (1998). Attention and eye movement in young infants: neural control and development. In J. E. Richards (Ed.), Cognitive neuroscience of attention: A developmental perspective (pp. 131-162). Mahwah, NJ: Erlbaum.

Rothbart, M. K., Ahadi, S. A., Hershey, K., \& Fisher, P. (1996). Investigations of temperament at 3-7 years: The children $s$ behavior questionnaire. Manuscript submitted for publication.

RuFf, H., \& RothbaRT, M. K. (1996). Attention in early development: Themes and variations. New York: Oxford University Press.

Simion, F., Val.enza, E., Umiltà, C., \& Barba, B. D. (1995). Inhibition of return in newborns is temporo-nasal asymmetrical. Infant Behavior \& Development, 18, 189-194.

Simon, J. (1990). The effects of an irrelevant directional cue on human information processing. In R. W. Proctor, T. G. Reeve, et al. (Eds.), Stimulus-response compatibility: An integrated perspective (pp. 3186). Amsterdam: North-Holland.

Stroop, J. R. (1935). Studies of interference in serial verbal reactions. Experimental Psychology, 18, 643-662.

TAYLOR, T. L., \& KLEIN, R. M. (1998). On the causes and effects of inhibition of return. Psychonomic Bulletin \& Review, 5, 625-643.

\section{NOTES}

1. The experiment using 5-year-old subjects was actually run using a previous version of the game in which there was only one type of animal (a bear) that appeared on the screen.

2 . In the no-tone trials, the delay before the onset of the target matched that of the tone trials. 
3. The reason for this specific order was a theoretical hypothesis that we were testing, in addition to the simple validation of the battery. The idea being tested was whether training with one network could influence the operation of another attentional network. This is why the data for 14 of the subjects who went through the described alert task are presented here. The rest of the sample did not participate in this version of the alert task. The theoretical rationale and testing of this hypothesis are described elsewhere in Berger, Jones, Rothbart, and Posner (2000).

4. In order to follow the rules of the game, in the incompatible trials of this task, there is a need for higher order processing of inhibition and negation of the more natural response. This ability to exert inhibitory control on one's own behavior develops with age and seems to depend on temperamental individual differences, as illustrated by the correlation with the relevant CBQ scales found in Carlson (1997) and in our sample.

5. Parents filled in the Children Behavior Questionnaire (Rothbart, Ahadi, Hershey, \& Fisher, 1996). Effortful control was defined as the composite of low pleasure, attentional shifting, and inhibitory control.

(Manuscript received November 4, 1999; revision accepted for publication March 2, 2000.) 